\title{
Effects of Dexmedetomidine on Inflammatory Factors and Mitogen Activated Protein Kinase-Related Signaling Pathways of Alveolar Lavage Fluid in Mice with Lipopolysaccharide-Induced Acute Lung Injury
}

\author{
HUI XIAO, S. LI, JIE YANG, HONGMEI YAO AND J. ZHENG* \\ Department of Anesthesiology, The First People's Hospital of Longquanyi District, Chengdu 610100, Sichuan Province, China
}

Xiao et al.: Effects of Dexmedetomidine in Mice with Induced Acute Lung Injury

To investigate the effects and mechanisms of Dexmedetomidine on inflammatory factors and mitogenactivated protein kinase related signaling pathways in alveolar lavage fluid of mice with acute lung injury induced by lipopolysaccharide. 36 clean-grade male Kunming mice were randomly divided into blank control group (normal control group), lipopolysaccharide-induced acute lung injury model group, and lipopolysaccharide+dexmedetomidine group, with 12 mice in each group. The

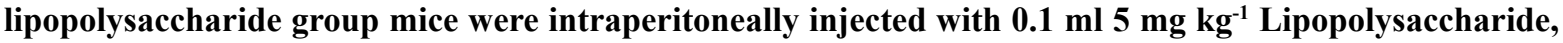
lipopolysaccharide+Dexmedetomidine mice were injected with $25 \mu \mathrm{g} \mathrm{kg}^{-1}$. Dexmedetomidine intraperitoneally with $0.1 \mathrm{ml}$ and $1 \mathrm{~h}$ later with $5 \mathrm{mg} \mathrm{kg}$. Lipopolysaccharide injected with $0.1 \mathrm{ml}$ intraperitoneally and the blank control group mice were intraperitoneally injected with normal saline of the same volume as the lipopolysaccharide group. All mice in each group were sacrificed after intraperitoneal injection for $6 \mathrm{~h}$, and their lung tissues were separated by thoracotomy and their bronchoalveolar lavage fluid was recovered for subsequent detection. Analysis groups of mice lung tissue pathology, compare groups of mice lung tissue pathology score, lung wet weight/dry weight (W)/(D), mitogen-activated protein kinase pathways (p38 lightning, extracellular signal regulating kinase, c-jun amino terminal kinase) and bronchoalveolar lavage fluid protein expression level of tumor necrosis factor alpha beta, interleukin-1ß, myeloperoxidase, protein concentration. In the blank control group, lung tissue structure was basically normal and alveolar structure was intact. lipopolysaccharide group mice had obvious lung tissue damage, and inflammatory cells appeared in the alveoli, with significantly increased exudation of red blood cells and proteins. Lipopolysaccharide+Dexmedetomidine group mice lung tissue damage significantly improved, inflammatory cell infiltration decreased, and red blood cell, protein exudation significantly decreased. Lung histopathological scores and bronchoalveolar lavage fluid levels of tumor necrosis factor alpha, interleukin$1 \beta$ and myeloperoxidase in lipopolysaccharide group were significantly higher than those in blank control group $(\mathbf{p}<\mathbf{0 . 0 5})$. The lung histopathological scores and the levels of tumor necrosis factor alpha, interleukin$1 \beta$ and myeloperoxidase in bronchoalveolar lavage fluid of lipopolysaccharide+Dexmedetomidine group were significantly lower than those of lipopolysaccharide group $(p<0.05)$. The protein concentration in W/D and bronchoalveolar lavage fluid in lipopolysaccharide group was significantly higher than that in blank control group $(\mathbf{p}<\mathbf{0 . 0 5})$. Protein concentration in $W / D$ and bronchoalveolar lavage fluid of lipopolysaccharide+Dexmedetomidine group was significantly lower than that of lipopolysaccharide group $(\mathbf{p}<\mathbf{0 . 0 5})$. The protein expression levels of extracellular signal regulating kinase, c-jun amino terminal kinase and P-P38 in the lipopolysaccharide group were significantly higher than those in the blank control group $(\mathbf{p}<\mathbf{0 . 0 5})$. The protein expression levels of extracellular signal regulating kinase, c-jun amino terminal kinase and P-P38 in the lipopolysaccharide+Dexmedetomidine group were significantly lower than those in the lipopolysaccharide group $(p<0.05)$. There was no significant difference in extracellular signal regulating kinase, c-jun amino terminal kinase and p38 protein expression levels in each group $(p>0.05)$. Dexmedetomidine can significantly alleviate acute lung injury induced by lipopolysaccharide in mice, and its mechanism may be realized by blocking the activation of mitogen-activated protein kinaserelated signaling pathway and thereby reducing inflammatory response, providing a new target for the treatment of sepsis induced acute lung injury in the futurey.

Key words: Acute lung injury, dexmedetomidine, mitogen-activated protein kinase, lipopolysaccharide, myeloperoxidase 
Acute lung injury is mainly manifested as intractable hypoxemia, respiratory distress and other symptoms. Although the assessment and treatment techniques of acute lung injury have been improved, the incidence of acute lung injury is still increasing year by year in recent years, and no effective drugs have been found to treat acute lung injury ${ }^{[1]}$. Sepsis and other causes can all induce the onset of acute lung injury, especially sepsis, which is the main cause of acute lung injury ${ }^{[2]}$. However, the pathological process of acute lung injury is extremely complex, and its pathogenesis has not been fully revealed. Therefore, it is of great significance to explore the pathogenesis of acute lung injury in order to improve the clinical symptoms and prognosis of patients. Before clinical study showed that excessive inflammatory cytokines secretion can participate in the pathological process of acute lung injury and play an important role in the Tumor necrosis factor- $\alpha$ (TNF- $\alpha$ ), Interleukin $1 \beta \quad$ (IL-1 $\beta)$, Interleukin-6 (IL-6), Myeloperoxidase, Myeloperoxidase (MPO) all belong to former inflammatory factor, can activate or enhance body inflammatory reaction ${ }^{[3,4]}$. Mitogen activated protein kinase (MAPK), which is widely present in many types of cells, can be activated under the induction of many factors such as ultraviolet light, inflammatory mediators and so on, and transfer the extracellular information to the nucleus, finally leading to a series of biological changes ${ }^{[5]}$. Dexmedetomidine (Dex) is currently widely used in critically ill patients, with ideal sedative and analgesic effects, and can be used as one of the adjuvant clinical anesthesia drugs ${ }^{[6]}$. Clinical studies have confirmed that dexmedetomidine plays an important role in resisting inflammatory response and protecting organs ${ }^{[7]}$. However, the mechanism of the anti-inflammatory effect of dex and sepsis induced acute lung injury is not clear, so the purpose of this study is to explore the effect of DEX on alveolar lavage fluid inflammatory factor and MAPK-related signaling pathway in mice with acute lung injury induced by lipopolysaccharide (LPS). 36 clean grade male Kunming mice were purchased from Shanghai Laboratory Animal Center of the Chinese Academy of Sciences with production license No.: SCXY (Shanghai) 2019-0005 and body mass (23 \pm 2$) \mathrm{g}$. All the 36 mice were raised in cages, 9 in each cage, feeding and drinking freely, changing day and night every $12 \mathrm{~h}$, the temperature was $25 \sim 30^{\circ}$, the humidity was $55 \sim 60^{\circ}$, and they were raised according to SPF grade. Dexmedetomi was ordered from Nanjing Saihongrui Biotechnology

*Address for correspondence E-mail: feian766400@163.com
This is an open access article distributed under the terms of the Creative Commons Attribution-NonCommercial-ShareAlike 3.0 License, which allows others to remix, tweak, and build upon the work non-commercially, as long as the author is credited and the new creations are licensed under the identical terms

Accepted 28 February 2021

Revised 02 September 2020

Received 15 March 2020

Indian J Pharm Sci 2021;83(1):153-157

Co., LTD. Lipopolysaccharide was purchased from was purchased from Youkang Hengye Biotechnology (Beijing) Co., LTD. ELISA kit was purchased from Guangzhou Jianlun Biotechnology Co., LTD. BCA protein detection kit was purchased from Shanghai Jingke Chemical Technology Co., LTD. Inverted microscope was purchased from Guangzhou Minmei Photoelectric Technology Co., LTD. Electronic balance purchased from Sedris Group, Germany; The marker (Shanghai) Co., LTD. The cryogenic high speed centrifuge was purchased from Beijing Ganming Gene Technology Co. LTD. 36 mice were randomly divided into the blank control group, LPS group and LPS+Dex group, with 12 mice in each group. Among them, the LPS group mice were intraperitoneally injected with 5 and the blank control group mice were intraperitoneally injected with normal saline of the same volume as the LPS group. Each group was intraperitoneal injection lavage fluid (BALF). He staining was used to detect and analyze the pathological changes of lung tissues of mice in each group. According to relevant literature ${ }^{[8]}$, the pulmonary histopathological scores of mice in each hemorrhage. The scores were $0 \sim 4$ points, 0 points: no damage occurred. 1 point: slight damage occurs;2 poisoned and damaged, and the total score ranged from BALF of mice in each group were detected by ELISA, and the protein level in BALF of mice in each group was detected by BCA protein kit. The middle lobe of the right lung of mice in each group was selected, and the exudate and blood stains on the surface were sucked dry and weighed on an electronic balance, which was the wet weight; After baking the middle lobe of the

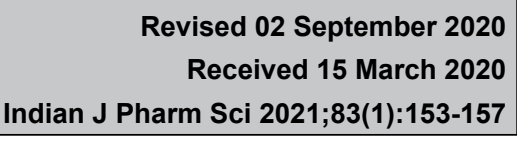

January-February 2021 0 to 12 . The contents of TNF- $\alpha$, IL- $1 \beta$ and MPO in 
right lung in the oven for $84 \mathrm{~h}$, it was taken out and weighed, which was the dry weight. The wet lung tissue weight (W)/dry lung tissue weight (D) was used to determine the degree of pulmonary tissue edema in each group. Western blot was used to detect the protein expression levels of MAPK-related pathways (P38, ERK and C-Jun n-terminal kinase (JNK)) in lung tissues of mice in each group. All data on this study adopts SPSS19.0 were analyzed, and the type of histopathological grading and BALF of TNF- $\alpha$, IL-1 $\beta$, MPO content measurement data such as adopt $(\overline{\mathrm{X}} \pm \mathrm{s})$ said, comparison between the two groups using $t$ test, multiple sets of comparison between using single factor analysis of variance, $\mathrm{p}<0.05$ as difference is significant. In the blank control group, lung tissue structure was basically normal and alveolar structure was intact. LPS group mice had obvious lung tissue damage, and inflammatory cells appeared in the alveoli, with significantly increased exudation of red blood cells and proteins. LPS+Dex group mice lung tissue damage significantly improved, inflammatory cell infiltration decreased, and red blood cell, protein exudation significantly decreased. $\mathrm{ABC}$ is shown in fig. 1. Lung histopathological scores and BALF levels of TNF- $\alpha$, IL- $1 \beta$ and MPO in LPS group were significantly higher than those in blank control group $(p<0.05)$. The lung histopathological scores and the levels of TNF- $\alpha$, IL-1 $\beta$ and MPO in BALF of LPS +Dex group were significantly lower than those of LPS group $(\mathrm{p}<0.05)$ (Table 1$)$. The protein concentration in W/D and BALF in LPS group was significantly higher than that in blank control group $(\mathrm{p}<0.05)$. Protein concentration in W/D and BALF of LPS+Dex group was significantly lower than that of LPS group $(\mathrm{p}<0.05)$ is shown in Table 2. The protein expression levels of P-ERK, P-JNK and P-P38 in THE LPS group were significantly higher than those in the blank control group $(\mathrm{p}<0.05)$. The protein expression levels of P-ERK, P-JNK and P-P38 in the LPS+Dex group were significantly lower than those in the LPS group $(\mathrm{p}<0.05)$. There was no significant difference in ERK, JNK and p38 protein expression levels in each group ( $p>0.05)$ is shown in Table 3 and fig. 2. Sepsis caused by cell infection can induce acute lung injury, and its morbidity and mortality are extremely high. Clinical research proves, systemic inflammatory response, anti inflammation disorders in the pathogenesis of acute lung injury, however the specific mechanism is not fully revealed, and the clinical effective interventions have not been discovered, so to reveal the pathogenesis of acute lung injury in order to improve patients is the mainstream of the current clinical staff pursuit direction ${ }^{[9]}$. Dexmedetomidine, as a short-term sedative, has little effect on respiratory function $^{[10]}$. The patient is easily aroused. Clinical literature has shown that dexmedetomidine can significantly inhibit sepsis and increase the expression level of inflammatory mediators in patients, which provides reference value for clinical treatment of sepsis ${ }^{[1]]}$. It has also been reported that dexmedetomidine was used to intervene in acute lung injury caused by
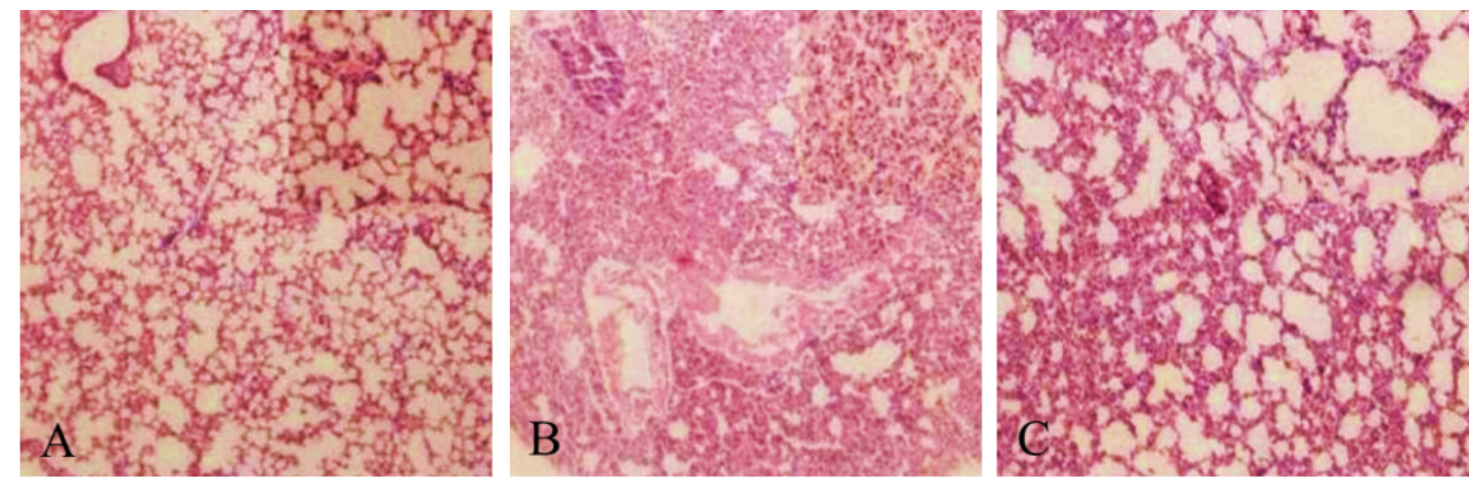

Fig. 1: Analysis of pathological changes in lung tissues of mice in each group

Note: A: Blank control group; B: LPS group; C: LPS+ Dex group

TABLE 1: NONHISTOPATHOLOGICAL SCORES AND BALF LEVELS OF TNF-A, IL-1B AND MPO IN EACH GROUP (X̄ $\pm S)$

\begin{tabular}{lccccc}
\hline Group & $\mathrm{n}$ & $\begin{array}{c}\text { Lung histopathological } \\
\text { Scores (score) }\end{array}$ & TNF-a (nmol/L) & IL-6 ( $\mu$ mol/L) & MPO (U/gprot) \\
\hline Control group & 12 & $1.05 \pm 0.02$ & $181.11 \pm 15.12$ & $31.36 \pm 4.31$ & $17.49 \pm 3.62$ \\
LPS group & 12 & $10.18 \pm 0.24^{*}$ & $302.33 \pm 23.66^{*}$ & $68.48 \pm 5.91^{*}$ & $31.36 \pm 2.49^{*}$ \\
LPS+ Dex group & 12 & $5.88 \pm 0.11^{\#}$ & $265.08 \pm 30.38^{\#}$ & $51.02 \pm 10.63^{\#}$ & $20.47 \pm 3.96^{\#}$ \\
\hline
\end{tabular}

Note: * indicates compared with the blank control group $p<0.05$; \# indicates compared to the LPS group $p<0.05$ 
TABLE 2: COMPARISON OF PROTEIN LEVELS IN W/D AND BALF IN EACH GROUP

\begin{tabular}{llll}
\hline Group & $\mathrm{n}$ & W/D & $\begin{array}{l}\text { Protein concentration } \\
\mathbf{( \% )}\end{array}$ \\
\hline Control group & 12 & $2.40 \pm 0.22$ & $108.70 \pm 20.21$ \\
LPS group & 12 & $5.99 \pm 0.24^{*}$ & $360.60 \pm 11.01^{*}$ \\
LPS+Dex group & 12 & $3.51 \pm 0.37^{\#}$ & $10.82 \pm 15.70^{*}$ \\
\hline
\end{tabular}

Note: * indicates compared with the blank control group $p<0.05$; \# indicates compared to the LPS group $p<0.05$

TABLE 3: COMPARISON OF PROTEIN EXPRESSION LEVELS IN MAPK-RELATED PATHWAYS OF MICE IN EACH GROUP

\begin{tabular}{lcccc}
\hline Group & $\mathrm{n}$ & $\mathrm{p}-\mathrm{ERK} / \mathrm{ERK}$ & $\mathrm{p}-\mathrm{JNK} / \mathrm{JNK}$ & $\mathrm{p}-\mathrm{p} 38 / \mathrm{p} 38$ \\
\hline $\begin{array}{l}\text { Control } \\
\text { group }\end{array}$ & 12 & $100.02 \pm 10.11$ & $100.00 \pm 2.15$ & $103.05 \pm 3.15$ \\
$\begin{array}{l}\text { LPS group } \\
12\end{array}$ & $762.59 \pm 15.89^{*}$ & $126.05 \pm 2.00^{*}$ & $184.13 \pm 3.01^{*}$ \\
$\begin{array}{l}\text { LPS+Dex } \\
\text { group }\end{array}$ & 12 & $180.05 \pm 14.05^{\#}$ & $104.05 \pm 2.15^{\#}$ & $108.98 \pm 3.27^{\#}$ \\
\hline $\begin{array}{l}\text { Note: * indicates p<0.05 compared with the blank control group; \# } \\
\text { indicates } \mathrm{p}<0.05 \text { compared to the LPS group }\end{array}$
\end{tabular}

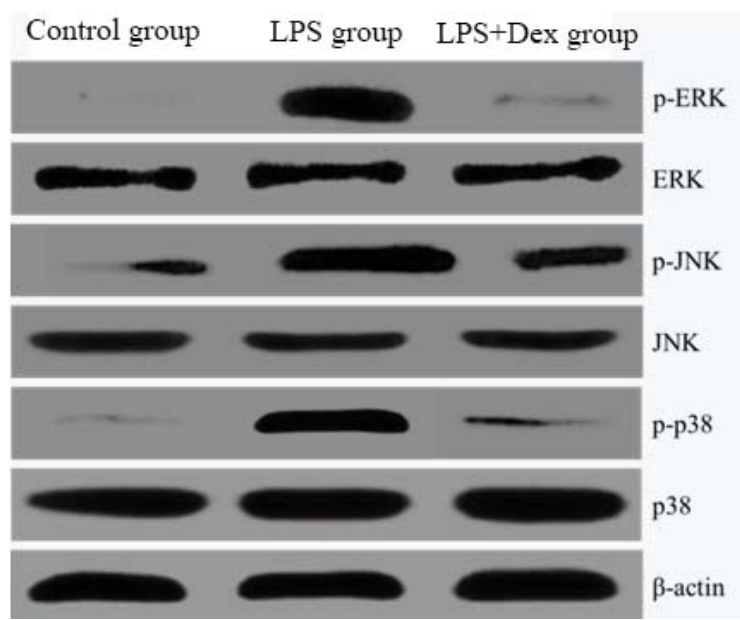

Fig. 2: Comparison of protein expression levels in MAPKrelated pathways of mice in each group

limb ischemia-reperfusion in rats, and it was found that the inflammatory response in rats was significantly alleviated $^{[12]}$. Clinical studies have confirmed that dexmedetomidine can significantly reduce the degree of ovarian tissue ischemia reperfusion injury. Studies on liver ischemia-reperfusion injury have shown that dexmedetomidine can play a protective role against erythrocyte deformability changes. In addition, dexmedetomidine can alleviate lung injury caused by pulmonary ischemia reperfusion ${ }^{[13]}$. Many foreign literatures have shown that in clinical practice, the use of dexmedetomidine in sedative sepsis patients can significantly reduce the production of inflammatory mediators, which is conducive to the alleviation of inflammatory responses and the extension of patient survival time ${ }^{[14]}$. All the above studies have confirmed that dexmedetomidine can play an anti-inflammatory and organ protective role. In this study, dexmedetomidine was used to intervene in mice with acute lung injury induced by LPS, and it was found that dexmedetomidine could significantly alleviate histopathological changes in mice with acute lung injury, reduce inflammatory cell infiltration, and significantly reduce erythrocyte and protein exudation. Clinical studies have confirmed that TNF- $\alpha$ and IL-1 $\beta$ can mediate the pneumoniamediated response in acute lung injury/acute respiratory distress syndrome. The lung hiathological scores and BALF levels of TNF- $\alpha$, IL-1 $\beta$, MPO, protein concentration and W/D in LPS+ Dex group were significantly lower than those in LPS group $(p<0.05)$. The results showed that dexmedetomidine could significantly reduce the release of TNF- $\alpha$ and other preinflammatory factors in bronchoalveolar lavage fluid of mice with acute lung injury, and improve the state of pulmonary edema. MAPK signaling pathways mainly include ERK, JNK and P38 signaling pathways, which are of great significance for maintaining normal physiological functions of cells, and the three pathways are interrelated to form a complex network ${ }^{[15]}$. Clinical studies have shown that LPS can participate in the pathogenesis of acute lung injury in sepsis and induce inflammatory responses. LPS can bind to cell membrane receptors and initiate signal transduction to the system, thereby activating the MAPK signaling pathway and ultimately accelerating the transcription of related genes to release many inflammatory mediators. The results of this study showed that the protein expression levels of P-ERK, P-JNK and P-P38 in the LPS+Dex group were significantly lower than those in the LPS group $(p<0.05)$. This suggests that dexmedetomidine may reduce inflammatory responses in mice with acute lung injury by inhibiting MAPK-related signaling pathways. In summary, Dex can significantly alleviate acute lung injury induced by LPS in mice, and its mechanism may be realized by blocking the activation of MAPK-related signaling pathway and thereby reducing inflammatory response, providing a new target for the treatment of sepsis induced acute lung injury in the future.

\section{Author's contribution:}

Hui Xiao and S. Li contributed equally to this work as co-first author.

\section{REFERENCES}

1. Wang BQ, Shi M, Zhang JP, Wu X, Chang MJ, Chen ZH, et al. Knockdown of TFPI-anchored endothelial cells exacerbates lipopolysaccharide-induced acute lung injury via NF-кB 
signaling pathway. Shock 2019;51(2):235-46.

2. Lee H, Kong G, Tran Q, Kim C, Park J, Park J. Relationship between ginsenoside Rg3 and metabolic syndrome. Front Pharmacol 2020;11:130.

3. Lu Y, Atkins SJ, Fernando R, Trierweiler A, Mester T, Grisolia $\mathrm{AB}$, et al. CD34- orbital fibroblasts from patients with thyroidassociated ophthalmopathy modulate TNF- $\alpha$ expression in CD34+ fibroblasts and fibrocytes. Invest Ophthalmol Vis Sci 2018;59(6):2615-22.

4. Pilitsi E, Peradze N, Perakakis N, Mantzoros CS. Circulating levels of the components of the GH/IGF-1/IGFBPs axis total and intact IGF-binding proteins (IGFBP) 3 and IGFBP 4 and total IGFBP 5, as well as PAPPA, PAPPA2 and Stanniocalcin-2 levels are not altered in response to energy deprivation and/ or metreleptin administration in humans. Metabolism 2019;97:32-9.

5. Lee KC, Chen YL, Lin PY, Chuang WL. Ursolic acid-induced apoptosis via regulation of the PI3K/Akt and MAPK signaling pathways in Huh-7 cells. Molecules 2018;23(8):2016.

6. Weerink MA, Struys MM, Hannivoort LN, Barends CR, Absalom AR, Colin P. Clinical pharmacokinetics and pharmacodynamics of dexmedetomidine. Clin Pharmacokinet 2017;56(8):893-913.

7. Menser C, Smith H. Emergence Agitation and Delirium: Considerations for Epidemiology and Routine Monitoring in Pediatric Patients. Local Reg Anesth 2020;13:73-83.

8. Sakaguchi M, Marutani E, Shin HS, Chen W, Hanaoka K, Xian M, et al. Sodium Thiosulfate Attenuates Acute Lung Injury in Mice. Anesthesiology 2014;121(6):1248-57.
9. Li R, Zhao L, Tong J, Yan Y, Xu C. Fine particulate matter and sulfur dioxide coexposures induce rat lung pathological injury and inflammatory responses via TLR4/p38/NF- $\mathrm{kB}$ pathway. Int J Toxicol 2017;36(2):165-73.

10. Guo Y, Gu D, Huang T, Cao L, Zhu X, Zhou Y, et al. Essential role of Salmonella Enteritidis DNA adenine methylase in modulating inflammasome activation. BMC Microbiol 2020;20(1):1-27.

11. Nie $\mathrm{Y}, \mathrm{Tu} \mathrm{W}$, Shen $\mathrm{X}, \mathrm{Yu} \mathrm{W}, \mathrm{Yu} \mathrm{Y}$, Song $\mathrm{X}$, et al. Dexmedetomidine Added to Sufentanil Patient-Controlled Intravenous Analgesia Relieves the Postoperative Pain after Cesarean Delivery: A Prospective Randomized Controlled Multicenter Study. Sci Rep 2018;8:9952-58.

12. Liu Y, Sheng B, Wang S, Lu F, Zhen J, Chen W. Dexmedetomidine prevents acute kidney injury after adult cardiac surgery: a meta-analysis of randomized controlled trials. BMC Anesthesiol 2018;18(1):1.

13. Song IK, Yi S, Lim HS, Lee JH, Kim EH, Cho JY, et al. A population pharmacokinetic model of intravenous dexmedetomidine for mechanically ventilated children after neurosurgery. J Clin Med 2019;8(10):1563-9.

14. Gu LF, Ge HT, Zhao L, Wang YJ, Zhang F, Tang HT, et al. Huangkui Capsule Ameliorates Renal Fibrosis in a Unilateral Ureteral Obstruction Mouse Model Through TRPC6 Dependent Signaling Pathways. Front Pharmacol 2020;11:996.

15. Kim EA, Kim SY, Ye BR, Kim J, Ko SC, Lee WW, et al. Anti-inflammatory effect of Apo-9'-fucoxanthinone via inhibition of MAPKs and NF-kB signaling pathway in LPSstimulated RAW 264.7 macrophages and zebrafish model. Int Immunopharmacol 2018;59:339-46. 